\title{
Pupillary Light Reflex is Altered in Adolescent Depression
}

\author{
A. MESTANIKOVA ${ }^{1,2}$, I. ONDREJKA $^{3}$, M. MESTANIK ${ }^{1,2}$, D. CESNEKOVA $^{3}$, \\ Z. VISNOVCOVA ${ }^{1,2}$, I. BUJNAKOVA ${ }^{1,2}$, M. OPPA ${ }^{3}$, A. CALKOVSKA ${ }^{1,2}$, \\ I. TONHAJZEROVA ${ }^{1,2}$
}

${ }^{1}$ Department of Physiology, Jessenius Faculty of Medicine in Martin, Comenius University in Bratislava, Martin, Slovakia, ${ }^{2}$ Biomedical Center Martin, Jessenius Faculty of Medicine in Martin, Comenius University in Bratislava, Martin, Slovakia, ${ }^{3}$ Psychiatric Clinic, Jessenius Faculty of Medicine in Martin, Comenius University in Bratislava, University Hospital Martin, Martin, Slovakia

Received December 16, 2016

Accepted February 26, 2017

\section{Summary}

Major depressive disorder is associated with abnormal autonomic regulation which could be noninvasively studied using pupillometry. However, the studies in adolescent patients are rare. Therefore, we aimed to study the pupillary light reflex $(P L R)$, which could provide novel important information about dynamic balance between sympathetic and parasympathetic nervous system in adolescent patients suffering from major depression. We have examined 25 depressive adolescent girls (age $15.2 \pm 0.3$ year) prior to pharmacotherapy and 25 age/gender-matched healthy subjects. PLR parameters were measured separately for both eyes after 5 min of rest using Pupillometer PLR-2000 (NeurOptics, USA). The constriction percentual change for the left eye was significantly lower in depressive group compared to control group (-24.12 $\pm 0.87 \%$ vs. $-28.04 \pm 0.96 \%, p<0.01)$. Furthermore, average constriction velocity and maximum constriction velocity for the left eye were significantly lower in depressive group compared to control group $(p<0.05, p<0.01$, respectively). In contrast, no significant between-groups differences were found for the right eye. Concluding, this study revealed altered PLR for left eye indicating a deficient parasympathetic activity already in adolescent major depression. Additionally, the differences between left and right eye could be related to functional lateralization of autonomic control in the central nervous system.

\section{Key words}

Major depressive disorder - Autonomic nervous system • Pupillary light reflex • Adolescent patients • Autonomic lateralization

\section{Corresponding author}

I. Tonhajzerova, Department of Physiology and Biomedical Center Martin, Jessenius Faculty of Medicine in Martin, Comenius University in Bratislava, Mala Hora 4C, 03601 Martin, Slovakia. E-mail: tonhajzerova@jfmed.uniba.sk or ingridtonhajzerova@gmail.com

\section{Introduction}

It is well-known that depressive disorder is associated with a deficient autonomic functioning associated with higher risk of cardiovascular adverse outcomes. For example, the studies revealed reduced cardiac vagal control and potential sympathetic overactivity using heart rate variability linear and nonlinear analysis in adolescent depressive patients (Tonhajzerova et al. 2010, Tonhajzerova et al. 2012). However, other authors found depression-linked abnormal autonomic control of different organs from cardiac modulation, e.g. gastric dysmotility caused by increased sympathetic modulation (Quick et al. 2010). In this context, the study of various effector organs innervated by autonomic nervous system has attracted more attention.

Pupillometry represents a noninvasive measurement of pupil diameter providing important information about dynamic balance between sympathetic and parasympathetic nervous system under central autonomic control. Specifically, the sympathetic branch, mediated by posterior hypothalamic nuclei, produces 
enlargement of the pupil by direct stimulation of the dilatator muscles. The contribution of the parasympathetic pathway is mediated by central inhibition of the Edinger-Westphal complex of the oculomotor nucleus in the midbrain as the motor center for parasympathetic pathway resulting in relaxation of the sphincter muscles and pupil dilatation (Steinhauer and Hakerem 1992). Taken together, the pupil size is determined by the tone of two muscles, the dilatator and the constrictor, thus, the pupillary dilatation can be the result of a stimulation of the dilatator regulated by sympathetic activity or an inhibition of constrictor innervated by parasympathetic nervous system (Laeng et al. 2012).

In this context, pupillary light reflex, i.e. pupil constriction in response to luminance increases, depends mainly on parasympathetic nervous system. Specifically, the afferent pathway from the retinal receptors involves only a synapse in the pretectum before secondary crossed and uncrossed projections reach the motor center in the third parasympathetic nerve - oculomotor nucleus. The efferent pathway involves a synapse at the ciliary ganglion, with the final neurons synapsing on pupillary sphincter to provide the major component of pupillary constriction (Steinhauer et al. 2000). Further, recent studies have demonstrated that pupillary response is included in psychological processes such as attention, cognitive and emotional processing (Binda et al. 2013, Jones et al. 2015, Stone et al. 2016), which abnormalities are associated with depression (Ottowitz et al. 2002). Therefore, the pathway for the pupillary light reflex provides an optimal model for investigating autonomic, cognitive and emotional influences in depression.

In adult age, the findings of pupillary responses in patients suffering from major depression are controversial. While previous studies revealed no significant differences in pupil diameter in depressive patients (Sokolski et al. 2000, Bär et al. 2004), recent studies demonstrated smaller pupillary responses in lessmotivated and anxious depressed participants (Jones et al. 2015), larger pupil area at the peak of constriction during pupillary light reflex in depressive patients (Wang et al. 2014) or positive correlation between pupil diameter stimulated by emotional sound and depressive score (Oguro et al. 2016). However, the childhood and adolescence could represent a specific vulnerable developmental period to pupillary light reflex parameters (Daluwatte et al. 2012) and potential depression-induced abnormal central autonomic control due to brain maturational changes (Yang et al. 2007, Thayer et al. 2009). Specifically, children and adolescents with major depression showed diminished pupil dilation to negative words (Silk et al. 2007); in contrast, already high risk youth with mothers' history of depression exhibit greater pupillary response to sad faces compared with children of nondepressed mothers (Burkhouse et al. 2014).

We addressed the hypothesis that adolescent major depressive disorder could be associated with autonomic dysfunction resulting in abnormal pupillary light reflex. Thus, the aim of this study is to examine the pupillary light reflex in patients suffering from major depression at adolescent age. To the best of our knowledge, it is the first study to assess central autonomic control using pupillary light reflex in adolescent depression.

\section{Methods}

\section{Subjects}

We have examined 25 adolescent girls with major depressive disorder (average age: $15.2 \pm 0.3$ year, body mass index $\left.22.5 \pm 0.8 \mathrm{~kg} / \mathrm{m}^{2}\right)$ and 25 healthy subjects matched for gender and age (control group). The patients suffering from MDD were recruited from the inpatients admitted to Psychiatric Clinic of Jessenius Faculty of Medicine and University Hospital in Martin. The diagnosis of severe depression, single episode without psychotic symptoms (e.g. mood congruent or incongruent delusions, hallucinations) and other comorbid psychiatric disorders (e.g. ADHD, anxiety disorders), was classified by thorough clinical investigation based on unstructured diagnostic interview by a staff child/adolescent psychiatrist according to Diagnostic and Statistical Manual of Mental Disorders, DSM-V (American Psychiatric Association 2013). The patients were examined before pharmacotherapy during the first week of hospitalization. The control participants have never been treated for any mental disorder. Following exclusion criteria were applied for both MDD and control group: underweight, overweight and obesity, history of cardiovascular, respiratory, endocrine, neurological, metabolic or infectious diseases, alcohol or drug abuse, smoking. Ophtalmologic screening indicated no ophtalmological diseases (other than correctable vision) in any of the subject. Moreover, all subjects were right-handed. 


\section{Ethics Statement}

The study was approved by the Ethics Committee of Jessenius Faculty of Medicine in Martin, Comenius University in Bratislava in accordance with the 1964 Helsinki declaration and its later amendments. All participants and their parents were carefully instructed about the study protocol and they gave written informed consent prior to examination.

\section{Study protocol}

All subjects were examined in a quiet darkened room with the same light intensity under standard conditions (temperature: $22-23{ }^{\circ} \mathrm{C}$ ) with minimization of stimuli in the morning between 8:00 and 11:30 a.m. after a normal breakfast. After $15 \mathrm{~min}$ of adaptation to the conditions of examination room the participants were instructed to lie comfortably in a special bed and rest in supine position for $5 \mathrm{~min}$. Then, the parameters of pupillary light reflex were assessed separately for both eyes using a handheld infrared optical scanner Pupillometer PLR-2000 (NeurOptics, USA) with a sampling frequency $32 \mathrm{~Hz}$ and accuracy $0.1 \mathrm{~mm}$. During the measurement the participants were asked to keep their head straight, fix their gaze on a spot located at a distance of $3 \mathrm{~m}$ and keep both eyes wide open without blinking. The pupillometer was kept at a right angle to the subject's axis of vision without tilting of the device. The margins of the pupil were electronically detected under infrared illumination and tracked during $5 \mathrm{~s}$ of continuous recording of the pupil after application of light stimulus with intensity of 180 microwatts and duration 154 milliseconds.

\section{Evaluated parameters}

The diameter of the pupil in millimeters was assessed before the application of light stimulus (initial value, INIT) and after illumination at the peak of the constriction (final value, END). The constriction amplitude in millimeters was calculated as INIT - END, and the percentual change (DELTA) of the pupil diameter during constriction was calculated as

$$
\text { DELTA }=(\text { INIT }- \text { END }) / \text { INIT } \times 100 \% .
$$

In addition, following parameters of the dynamics of pupillary light reflex were assessed: the time of the onset of the constriction after application of light stimulus (latency, LAT), average constriction velocity $(\mathrm{ACV}, \mathrm{mm} / \mathrm{s})$, maximum constriction velocity $(\mathrm{MCV}$, $\mathrm{mm} / \mathrm{s}$ ), and average dilatation velocity after having reached the peak of the constriction $(\mathrm{ADV}, \mathrm{mm} / \mathrm{s})$.

\section{Statistical analysis}

The data were analysed using statistical software package SYSTAT 10 (Cranes Software International Ltd, USA). The non-Gaussian/Gaussian distribution was ascertained by Shapiro-Wilk normality test and all the data showed Gaussian distribution. Consecutively, Student's unpaired test was used for between-groups comparison and Student's paired test for comparison of the side differences. The probabilities $\mathrm{p}<0.05$ were considered as significant. The data are expressed as mean \pm SEM.

\section{Results}

\section{Between-groups comparisons}

Regarding the left eye, the parameter DELTA representing the constriction percentual change was significantly lower in depressive group compared to control group $(-24.12 \pm 0.87 \%$ vs. $-28.04 \pm 0.96 \%$, $<<0.01)$. Furthermore, average constriction velocity $(\mathrm{ACV})$ and maximum constriction velocity (MCV) were significantly shortened in depressive group compared to control group for the left eye $(p<0.05, p<0.01$, respectively). No significant differences were found in the remaining parameters - initial diameter (INIT), final diameter (END), latency (LAT) and average dilatation velocity (ADV) for left eye. In contrast, statistical analysis revealed no significant between-groups differences in parameters of pupillary light reflex for the right eye (Table 1).

\section{Side differences}

No significant differences between the left and right eyes were found in parameters of pupillary light reflex in alone depressive and control groups.

\section{Discussion}

In this study, we assessed central autonomic activity using pupillary light reflex (PLR) in adolescent major depression. The major findings of this study are following: 1) the pupillary light reflex was diminished in adolescent patients suffering from depressive disorder; 2) the pupil response to light was different between left and right eye in adolescent major depression compared to controls. It seems that discrete abnormalities in central autonomic integrity resulting in atypical PLR pattern for left eye could characterize major depression already in adolescent age-period. 
Table 1. Parameters of the pupillary light reflex.

\begin{tabular}{|c|c|c|c|}
\hline & Controls $(n=25)$ & $\operatorname{MDD}(n=25)$ & $\mathrm{p}$-value \\
\hline \multicolumn{4}{|l|}{ Left eye } \\
\hline $\operatorname{INIT}(\mathrm{mm})$ & $4.57 \pm 0.18$ & $4.51 \pm 0.25$ & 0.837 \\
\hline$E N D(\mathrm{~mm})$ & $3.28 \pm 0.13$ & $3.41 \pm 0.19$ & 0.586 \\
\hline Constriction (mm) & $1.29 \pm 0.07$ & $1.10 \pm 0.08$ & 0.074 \\
\hline DELTA $(\%)$ & $-28.04 \pm 0.96$ & $-24.12 \pm 0.87$ & 0.004 \\
\hline $\operatorname{LAT}(s)$ & $0.21 \pm 0.01$ & $0.27 \pm 0.05$ & 0.274 \\
\hline$A C V(\mathrm{~mm} / \mathrm{s})$ & $-3.77 \pm 0.14$ & $-3.25 \pm 0.15$ & 0.014 \\
\hline $\operatorname{MCV}(\mathrm{mm} / \mathrm{s})$ & $-4.82 \pm 0.19$ & $-4.02 \pm 0.19$ & 0.005 \\
\hline$A D V(\mathrm{~mm} / \mathrm{s})$ & $1.57 \pm 0.07$ & $1.39 \pm 0.09$ & 0.149 \\
\hline \multicolumn{4}{|l|}{ Right eye } \\
\hline INIT (mm) & $4.50 \pm 0.18$ & $4.51 \pm 0.28$ & 0.981 \\
\hline$E N D(m m)$ & $3.24 \pm 0.13$ & $3.33 \pm 0.21$ & 0.711 \\
\hline Constriction (mm) & $1.26 \pm 0.08$ & $1.18 \pm 0.10$ & 0.503 \\
\hline DELTA $(\%)$ & $-28.00 \pm 1.17$ & $-25.56 \pm 1.19$ & 0.149 \\
\hline$L A T(s)$ & $0.21 \pm 0.01$ & $0.22 \pm 0.01$ & 0.484 \\
\hline$A C V(\mathrm{~mm} / \mathrm{s})$ & $-3.68 \pm 0.16$ & $-3.25 \pm 0.18$ & 0.083 \\
\hline$M C V(\mathrm{~mm} / \mathrm{s})$ & $-4.65 \pm 0.21$ & $-4.09 \pm 0.24$ & 0.087 \\
\hline$A D V(\mathrm{~mm} / \mathrm{s})$ & $1.49 \pm 0.07$ & $1.35 \pm 0.09$ & 0.210 \\
\hline
\end{tabular}

MDD - major depressive disorder, INIT - diameter of the pupil before the application of light stimulus, END - diameter of the pupil at the peak of the constriction, Constriction - constriction amplitude, DELTA - percentual change of the diameter of the pupil, LAT - time of the constriction onset after application of light stimulus, ACV - average constriction velocity, MCV - maximum constriction velocity, ADV - average dilatation velocity. Values are expressed as mean \pm SEM. Probability $\mathrm{p}<0.05$ was considered as significant.

Specifically, the parasympathetic subdivision plays a dominant role during the pupil constriction phase, and both parasympathetic and sympathetic nervous system innervate the pupil in the beginning of the PLR redilatation phase. In this context, reduced parasympathetic activity is characterized by PLR longer constriction latency, slower maximum constriction velocity and smaller constriction amplitude (Lowenstein and Loewenfeld 1950). Thus, our results of altered PLR parameters - smaller percentual change of constriction amplitude associated with slower average and maximum constriction velocity could indicate a deficient parasympathetic activity in adolescent major depression. These findings are in agreement with studies regarding depression-linked PLR abnormalities in adult depressive patients, and extend them into adolescent age-period. Moreover, the indices associated with dilatation phase expressing predominantly sympathetic activation (Wang et al. 2016) were without significant differences between depression and control groups. Several explanations are assumed.
Firstly, the primary circuit responsible for the pupillary constriction in response to light is subcortical, with the Edinger-Westphal nucleus receiving excitatory input from the pretectal olivary nucleus activated by retinal ganglion cells (Güler et al. 2008). Specifically, the light falling on the retina leads to increased neural activity in the pretectal regions, consequent activation of preganglionic parasympathetic neurons innervating the ciliary ganglion, and to the activation of the constrictor muscle resulting in pupil constriction (Thompson 1992, McDougal and Gamlin 2015). Therefore, the diminished PLR found in adolescent depressive girls could be explained by reduction of activity in preganglionic parasympathetic fibers and ciliary ganglion neurons. Moreover, the ciliary ganglion and constrictor muscles contain acetylcholine receptors. Acetylcholine as a principal neurotransmitter of the parasympathetic nervous system reacts rapidly, therefore, it is associated with the phase of constriction (Wang et al. 2016). Thus, reduced parasympathetic activity indexed by PLR measures could reflect depression-related 
cholinergic deficiency, as suggested in recent studies (Wang et al. 2014). It is important to note that this circuit is modulated by several inputs, such as anatomical projections from the visual cortex and from the superior colliculus to the pretectal olivary nucleus and by receiving excitatory input from extrastriate visual areas (Binda et al. 2013). In this context, we could speculate that our finding of reduced parasympathetic-linked PLR control could be affected by the more widespread complex neuropathological abnormalities associated with major depression, e.g. altered neurotransmitter functioning within occipital cortex characterized by lower levels of gamma-aminobutyric acid as well as $\mathrm{N}$-acetylaspartate, and by increased concentrations of glutamate and glutamine (Bhagwagar et al. 2007).

Furthermore, the PLR is directly innervated by neural circuitry implicated in physiological arousal including subcortical brain structure locus coeruleus. Importantly, the Edinger-Westphal region receives inhibitory input from the locus coeruleus, and a reduction in this inhibitory input can result in pupil constriction (Wilhelm et al. 2002, Aston-Jones and Cohen 2005). In other words, the activity of parasympathetic oculomotor center is inhibited by the sympathetic-linked noradrenergic systems' locus coeruleus (Wilhelm et al. 1999). Therefore, the relative sympathetic dominance could represent one of potential pathomechanisms leading to abnormal pupillary light reflex in major depression: if sympathetic nervous activity is higher, the neural inhibitory mechanism on Edinger-Westphal nucleus will be excited resulting in pupillary dilatation (Laeng et al. 2012). This finding is in accordance with our previous study revealed significantly higher cardiac beta-adrenergic activity in adolescent major depression (Tonhajzerova et al. 2010). Taken together, diminished PLR in constriction phase might indicate relative dominance of central sympathetic control associated with parasympathetic underactivity at the level of subcortical centers in adolescent depressive patients.

Notably, the parasympathetic center for pupillary constriction - Edinger-Westphal region is also modulated by inhibitory influences from descending cortical pathways (Steinhaer and Hakerem 1992). In particular, cognitive load associated with prefrontal and frontal cortical functioning contributes to this inhibitory process mediated by direct cortical and indirect corticothalamic-hypothalamic pathways resulting in inhibition of the Edinger-Westphal region. Moreover, the general increase in inhibition during a sustained performance of difficult task may include contributions of reticular pathways contributing to arousal, which also affect the Edinger-Westphal complex (Steinhauer et al. 2004). Recent studies showed abnormal functioning of prefrontal cortical activity resulting in deficient inhibitory influence on parasympathetic oculomotor complex in depressive patients (Siegle et al. 2011); therefore, it might represent a potential mechanism leading to diminished PLR found in our depressive patients.

Further, potential brain mechanisms of altered pupillary light reflex in adolescent depression could include impaired reactivity in emotional circuitry, such as amygdala or anterior cingulate cortex (Laeng et al. 2012). Several recent studies showed diminished pupil dilatation to negative words in children with major depression (Silk et al. 2007), or increased pupil dilatation to sad faces in children of mothers with a history of major depression compared to children of nondepressed mothers (Burkhouse et al. 2014). Moreover, objective measurements based on concurrent pupillary and functional magnetic resonance imaging studies suggest that pupil response provides information related to brain structures included in cognitive-affective regulation (Siegle et al. 2003). Taken together, it seems that complex central network consisting of multiple brain regions and pathways implicated in cognitive-affective regulation could affect pupillary response to light in major depression.

It is important to note that the PLR parameters associated with constriction phase (constriction ratio, maximum constriction acceleration and velocity) positively correlated with heart rate variability (HRV) measures indicating cardiac vagal activity (HF-HRV, rMSSD, pNN50) in healthy subjects (Kaltsatou et al. 2011). In this context, our previous studies revealed impaired complex cardiac vagal control indexed by linear as well as nonlinear HRV measures in adolescent major depression (Tonhajzerova et al. 2009, Tonhajzerova et al. 2010, Tonhajzerova et al. 2012). It seems that central brain control areas for pupil and heart autonomic regulation are mutually interconnected. Specifically, the central autonomic network (Benarroch 1993) as a highly integrated system regulating complex cardiac integrity includes the brain areas associated with both cognitive and emotional processing, such as prefrontal cortex, anterior cingulate cortex or amygdala which also play an important role in control of pupillary responses (Laeng et al. 2012). Thus, we assume that adolescent major depression could be characterized by abnormal complex 
central autonomic regulation in the pattern of parasympathetic-linked underactivity with potential sympathetic dominance resulting in abnormal peripheral effectors' responses. Further research in this field is needed.

Interestingly, despite the fact that differences between the left and right eye were not found in alone control or depressive group, only PLR parameters from the left eye significantly differed between groups depression vs. controls. This finding could be related to the effect of hemispheric lateralization of cortical autonomic control where parasympathetic activity is considered to be under dominant influence of the left hemisphere, as found in the studies of cardiovascular autonomic regulation (Wittling et al. 1998, Hilz et al. 2001, Guo et al. 2016). However, it is not clear if the same applies also for the laterality of the autonomic control of the PLR. Importantly, Bär et al. (2005) found that parasympathetic parameters of the PLR were more pronounced for the right in contrast to the left eye. Therefore, it is unclear whether our findings of reduced parasympathetic activity in depressive group indexed by the PLR measures (MCV, DELTA, ACV) from the left eye could reflect an alteration of the autonomic modulatory pathway from the left hemisphere, or whether it could indicate greater sensitivity of the left eye to discrete autonomic abnormalities compared to parasympathetically-dominated right eye. Future research based on functional imaging methods might elucidate this issue.

Finally, the abnormal PLR could be related to subclinical and latent symptoms of anxiety in major depression. Recent studies concluded that arousal associated with anxiety contributes to inhibition of the pupillary light reaction. In particular, the patients with anxiety disorders have shown decreased pupillary responses to light compared to controls, though initial diameters did not differ (Bakes et al. 1990, Jones et al. 2015). Thus, it is questionable whether our results of diminished PLR indicate pure abnormalities in complex central autonomic network regulating pupil light reflex, or they are related to clinically asymptomatic and latent comorbid anxiety disorder in adolescent patients with major depression. Longitudinal study could help to resolve this question.

\section{Limitations}

Potential limitation of our study is a relatively small homogeneous group of female patients with major depressive disorder. From this point of view, the findings of this study cannot be extrapolated to general population and need to be independently validated in larger groups of patients with respect to gender differences.

\section{Conclusions}

Our study revealed diminished pupillary light reflex for left eye in girls suffering from major depression which could indicate abnormal depression-linked central parasympathetic control already in adolescent age. Additionally, the different results for left and right eye could be related to the effect of lateralization of cortical centers regulating autonomic nervous system.

\section{Conflict of Interest}

There is no conflict of interest.

\section{Acknowledgements}

This work was supported by VEGA 1/0087/14 and the project „Biomedical Center Martin“ ITMS code: 26220220187, co-financed from EU sources.

\section{References}

AMERICAN PSYCHIATRIC ASSOCIATION: Depressive disorders. In: Diagnostic and Statistical Manual of Mental Disorders DSM-5. Fifth Edition. American Psychiatric Association, Arlington, 2013, pp 155-188.

ASTON-JONES G, COHEN JD: An integrative theory of locus coeruleus-norepinephrine function: adaptive gain and optimal performance. Annu Rev Neurosci 28: 403-450, 2005.

BAKES A, BRADSHAW CM, SZABADI E: Attenuation of the pupillary light reflex in anxious patients. $B r J C l i n$ Pharmacol 30: 377-381, 1990.

BÄR KJ, BOETTGER MK, TILL S, DOLICEK J, SAUER H: Lateralization of pupillary light reflex parameters. Clin Neurophysiol 116: 790-798, 2005. 
BÄR KJ, GREINER W, JOCHUM T, FRIEDRICH M, WAGNER G, SAUER H: The influence of major depression and its treatment on heart rate variability and pupillary light reflex parameters. $J$ Affect Disord 82: 245-252, 2004.

BENARROCH EE: The central autonomic network: functional organisation, dysfunction, and perspective. Mayo Clin Proc 68: 988-1001, 1993.

BHAGWAGAR Z, WYLEZINSKA M, JEZZARD P, EVANS J, ASHWORTH F, SULE A, MATTHEWS PM, COWEN PJ: Reduction in occipital cortex gamma-aminobutyric acid concentrations in medication-free recovered unipolar depressed and bipolar subjects. Biol Psychiatry 61: 806-812, 2007.

BINDA P, PEREVERZEVA M, MURRAY SO: Attention to bright surfaces enhances the pupillary light reflex. J Neurosci 33: 2199-2204, 2013.

BURKHOUSE KL, SIEGLE GJ, GIBB BE: Pupillary reactivity to emotional stimuli in children of depressed and anxious mothers. J Child Psychol Psychiatry 55: 1009-1016, 2014.

DALUWATTE C, MILES JH, CHRIST SE, BEVERSDORF DQ, LOFGREEN A, BERLINER N, YAO G: Age-dependent pupillary light reflex parameters in children. Conf Proc IEEE Eng Med Biol Soc 2012: 3776-3779, 2012.

GUO CC, STURM VE, ZHOU J, GENNATAS ED, TRUJILLO AJ, HUA AY, CRAWFORD R, STABLES L, KRAMER JH, RANKIN K, LEVENSON RW, ROSEN HJ, MILLER BL, SEELEY WW: Dominant hemisphere lateralization of cortical parasympathetic control as revealed by frontotemporal dementia. Proc Natl Acad Sci U S A 113: E2430-E2439, 2016.

GÜLER AD, ECKER JL, LALL GS, HAQ S, ALTIMUS CM, LIAO HW, BARNARD AR, CAHILL H, BADEA TC, ZHAO H, HANKINS MW, BERSON DM, LUCAS RJ, YAU KW, HATTAR S: Melanopsin cells are the principal conduits for rod-cone input to non-image-forming vision. Nature 453: 102-105, 2008.

HILZ MJ, DÜTSCH M, PERRINE K, NELSON PK, RAUHUT U, DEVINSKY O: Hemispheric influence on autonomic modulation and baroreflex sensitivity. Ann Neurol 49: 575-584, 2001.

JONES NP, SIEGLE GJ, MANDELL D: Motivational and emotional influences on cognitive control in depression: A pupillometry study. Cogn Affect Behav Neurosci 15: 263-275, 2015.

KALTSATOU A, KOUIDI E, FOTIOU D, DELIGIANNIS P: The use of pupillometry in the assessment of cardiac autonomic function in elite different type trained athletes. Eur J Appl Physiol 111: 2079-2087, 2011.

LAENG B, SIROIS S, GREDEBÄCK G: Pupillometry: a window to the preconscious? Perspect Psychol Sci 7: 18-27, 2012.

LOWENSTEIN O, LOEWENFELD IE: Mutual role of sympathetic and parasympathetic in shaping of the pupillary reflex to light. Arch Neurol Psychiatr 64: 341-377, 1950.

MCDOUGAL DH, GAMLIN PD: Autonomic control of the eye. Compr Physiol 5: 439-473, 2015.

OGURO H, SUYAMA N, KARINO K, YAMAGUCHI S: Pupillary response to auditory stimuli in depressive state. J Neurol Res 6: 8-11, 2016.

OTTOWITZ WE, DOUGHERTY DD, SAVAGE CR: The neural network basis for abnormalities of attention and executive function in major depressive disorder: implications for application of the medical disease model to psychiatric disorders. Harv Rev Psychiatry 10: 86-99, 2002.

QUICK C, KLIEM A, BERGER S, HOCKE M, TANCER M, JUCKEL G, YERAGANI VK, BÄR KJ: Gastric dysmotility in major depression. Prog Neuropsychopharmacol Biol Psychiatry 34: 92-97, 2010.

SIEGLE GJ, STEINHAUER SR, FRIEDMAN ES, THOMPSON WS, THASE ME: Remission prognosis for cognitive therapy for recurrent depression using the pupil: utility and neural correlates. Biol Psychiatry 69: 726-733, 2011.

SIEGLE GJ, STEINHAUER SR, STENGER VA, KONECKY R, CARTER CS: Use of concurrent pupil dilation assessment to inform interpretation and analysis of fMRI data. Neuroimage 20: 114-124, 2003.

SILK JS, DAHL RE, RYAN ND, FORBES EE, AXELSON DA, BIRMAHER B, SIEGLE GJ: Pupillary reactivity to emotional information in child and adolescent depression: links to clinical and ecological measures. Am J Psychiatry 164: 1873-1880, 2007.

SOKOLSKI KN, NGUYEN BD, DEMET EM: Decreases in dilated pupil size in depressed patients with age may reflect adrenergic changes. Psychiatry Res 94: 267-272, 2000. 
STEINHAUER SR, CONDRAY R, KASPAREK A: Cognitive modulation of midbrain function: task-induced reduction of the pupillary light reflex. Int J Psychophysiol 39: 21-30, 2000.

STEINHAUER SR, HAKEREM G: The pupillary response in cognitive psychophysiology and schizophrenia. Ann N Y Acad Sci 658: 182-204, 1992.

STEINHAUER SR, SIEGLE GJ, CONDRAY R, PLESS M: Sympathetic and parasympathetic innervation of pupillary dilation during sustained processing. Int J Psychophysiol 52: 77-86, 2004.

STONE LB, SILK JS, SIEGLE GJ, LEE KH, STROUD LR, NELSON EE, DAHL RE, JONES NP: Depressed adolescents' pupillary response to peer acceptance and rejection: the role of rumination. Child Psychiatry Hum Dev 47: 397-406, 2016.

THAYER JF, SOLLERS III JJ, LABINER DM, WEINAND M, HERRING AM, LANE RD, AHERN GL: Age-related differences in prefrontal control of heart rate in humans: a pharmacological blockade study. Int J Psychophysiol 72: 81-88, 2009.

THOMPSON HS: The pupil. In: Adler's Physiology of the Eye 9th.: Clinical Application. ADLER FH, HART WM (eds), Mosby Year Book, St. Louis, 1992, pp 412-441.

TONHAJZEROVA I, ONDREJKA I, CHLADEKOVA L, FARSKY I, VISNOVCOVA Z, CALKOVSKA A, JURKO A, JAVORKA M: Heart rate time irreversibility is impaired in adolescent major depression. Prog Neuropsychopharmacol Biol Psychiatry 39: 212-217, 2012.

TONHAJZEROVA I, ONDREJKA I, JAVORKA M, ADAMIK P, TURIANIKOVA Z, KERNA V, JAVORKA K, CALKOVSKA A: Respiratory sinus arrhythmia is reduced in adolescent major depressive disorder. Eur J Med Res 14: 280-283, 2009.

TONHAJZEROVA I, ONDREJKA I, JAVORKA K, TURIANIKOVA Z, FARSKY I, JAVORKA M: Cardiac autonomic regulation is impaired in girls with major depression. Prog Neuropsychopharmacol Biol Psychiatry 34: 613-618, 2010.

WANG J, FAN Y, ZHAO X, CHEN N: Pupillometry in Chinese female patients with depression: a pilot study. Int J Environ Res Public Health 11: 2236-2243, 2014.

WANG Y, ZEKVELD AA, NAYLOR G, OHLENFORST B, JANSMA EP, LORENS A, LUNNER T, KRAMER SE: Parasympathetic nervous system dysfunction, as identified by pupil light reflex, and its possible connection to hearing impairment. PLoS One 11: e0153566, 2016.

WILHELM BJ, WILHELM H, MORO S, BARBUR JL: Pupil response components: studies in patients with Parinaud's syndrome. Brain 125: 2296-2307, 2002.

WILHELM B, WILHELM H, LÜDTKE H: Pupillography: Principles and applications in basic and clinical research. In: Pupillography: Principles, Methods and Applications. KUHLMANN J, BOTTCHER M (eds), Zuckschwerdt Verlag, München, 1999, pp 1-11.

WITTLING W, BLOCK A, SCHWEIGER E, GENZEL S: Hemisphere asymmetry in sympathetic control of the human myocardium. Brain $\operatorname{Cogn}$ 38: 17-35, 1998.

YANG TT, SIMMONS AN, MATTHEWS SC, TAPERT SF, BISCHOFF-GRETHE A, FRANK GK, ARCE E, PAULUS MP: Increased amygdala activation is related to heart rate during emotion processing in adolescent subjects. Neurosci Lett 428: 109-114, 2007. 